\title{
El efecto enero en las principales bolsas latinoamericanas de valores*
}

\author{
Francisco López Herrera \\ Investigador, División de \\ Investigación, Facultad de \\ Contaduría y Administración, \\ UNAM. francisco_lopez_he- \\ rrera@yahoo.com.mx
}

\section{Domingo Rodríguez}

Benavides

Investigador, División de Posgrado, Facultad de Economía, UNAM.

*Los autores agradecen los comentarios y sugerencias de dos dictaminadores anónimos. Es responsabilidad exclusiva de los autores cualquier error $\mathrm{u}$ omisión que aún quede.

\section{Resumen}

El objetivo de este trabajo es ofrecer evidencia empírica sobre la presencia del denominado efecto enero, así como de otros efectos estacionales, en los principales mercados de capitales de Latinoamérica. Para tal fin, en el análisis se emplean dos especificaciones econométricas uniecuacionales con variables dummy para los rendimientos accionarios de cada mercado, valuados tanto en su moneda local como en dólares. Debido a que en la estimación de dichas especificaciones se detectaron efectos ARCH para algunos países, se amplió la especificación de las pruebas con un modelo GARCH para las volatilidades de los rendimientos de esos mercados. Se encuentra evidencia mixta sobre la existencia del efecto enero en dichos mercados la cual depende de: 1) la especificación con que se lleve a cabo la prueba, y 2) de la forma en que se valúen dichos rendimientos, ya sea en moneda local o en dólares.

Palabras clave: mercados accionarios emergentes, efecto enero, rendimientos accionarios, efectos estacionales, modelos GARCH.

Clasificación JEL: C22, G11 y G15. 


\title{
The january effect in the main Latin-American stock exchanges
}

\begin{abstract}
The aim of this paper is to provide empirical evidence about the presence of so-called January Effect, and other seasonal effects, in major Latin American capital markets. To this end, in the analysis two single-equation econometric specifications with dummy variables are used for stock returns in each market, valued both in local currency and dollars. In estimating such specifications ARCH effects were detected for some countries, so the test specification was expanded with a GARCH model for the volatility of the returns on these markets. In the end, mixed evidence on the existence of January effect is found in those markets, evidence which depends on: 1) the specification used in the test, and 2) how returns are valued, whether in local currency or in US dollars.
\end{abstract}

Keywords: emerging stock markets, january effect, stock returns, seasonal effects, GAR$\mathrm{CH}$ models.

JEL classification: C22, G11 and G15.

\section{Introducción}

El efecto enero es un fenómeno de patrón que se ha observado principalmente en los precios de las acciones, aunque también existe evidencia de la existencia de este efecto en el mercado de bonos. Dicho efecto consiste en una tendencia a rendimientos más elevados en el mercado de valores en enero, especialmente en las acciones de empresas pequeñas, dando lugar a un movimiento alcista generalizado. Es decir, por el efecto enero presumiblemente se producen mayores rentabilidades anormales positivas en ese mes que en el resto del año. Este hecho ha sido ampliamente documentado en los mercados de capitales de diversos países y se atribuye a diversas razones: un premio por tamaño para las empresas más pequeñas; la venta de acciones en diciembre para realizar pérdidas fiscales; o el maquillaje de los inversionistas institucionales que venden al final de año determinadas acciones con objeto de aparentar menor riesgo como lo sugieren Hai y Qingzhong (2003).

Otra de las explicaciones sobre la existencia del efecto enero estriba en que a partir de enero las empresas comienzan a difundir noticias de beneficios del ejercicio económico que acaba de terminar. Los resultados encontrados por Hai y Qingzhong (2003) sugieren la existencia de una relación entre el anuncio anual del 
resultado del ejercicio anterior y el efecto enero, por lo que la existencia de ciclos en las rentabilidades, en dicho mes, podría ser atribuida a los anuncios de variables fundamentales de la empresa, como las ganancias del ejercicio previo, rentabilidad y cambios en la situación financiera.

Independientemente de cuál es la causa del efecto enero, esta regularidad empírica ha producido interés en el ámbito académico puesto que su existencia, al igual que la de otros patrones como el efecto día de la semana o los patrones observables durante el día de operaciones, no sería congruente con el funcionamiento de un mercado eficiente en el cual se eliminarían tales patrones al ser detectados por los inversionistas. Es esta razón por la cual al efecto enero se le clasifica entre las llamadas anomalías de mercado.

Naturalmente que la existencia del efecto enero en un mercado resulta también de gran interés para los inversionistas, puesto que les permitiría diseñar estrategias de inversión para aprovechar los beneficios derivados de la presencia de ese efecto. En particular destaca que los llamados mercados emergentes se han convertido en objetivos de los inversionistas internacionales de portafolio, motivados por los procesos de liberalización que se han llevado a cabo en los países sede de tales mercados motivados por el afán de captar inversión extranjera de portafolio que complemente su ahorro interno. Aunque la importancia de estos mercados es mayor cada día, sigue siendo marginal el lugar que ocupan en la agenda de la investigación financiera, razón por la cual es necesario realizar más investigación sobre ellos.

Por lo anterior, el propósito de este trabajo es recabar evidencia sobre la presencia del efecto enero en los mercados latinoamericanos de capitales más importantes, es decir, los mercados de Argentina, Brasil, Chile, Colombia, México y Perú, mediante análisis empírico. El documento tiene la siguiente estructura: en la sección 1 se ofrece un recuento de los aspectos relevantes que han surgido en la literatura sobre el tema, la sección 2 se ocupa de presentar el modelo econométrico que se emplea en la sección 3 para llevar a cabo las estimaciones empíricas. Finalmente, se presentan las conclusiones.

\section{Aspectos relevantes de la literatura sobre el efecto enero}

En el mercado estadounidense se obtuvo primero evidencia empírica sobre la existencia de rendimientos más altos durante enero. En el estudio pionero de Rozeff y Kinney (1976) se reporta que de 1904 a 1974 el rendimiento promedio del merca- 
do bursátil de Nueva York era notoriamente más alto en enero, ocho veces, que el promedio para cualquier otro mes del año. Posteriormente Keim (1983) encontró que en enero eran más altos los promedios de los rendimientos diarios del NYSE y AMEX. Al estudiar el índice S\&P 500, Fama (1991) también encontró evidencia de la existencia del efecto enero. Los resultados de Keim (1989) sugieren que al menos una parte del efecto enero observado en los mercados de valores estadounidenses podría explicarse como consecuencia de tendencias sistemáticas inducidas por la forma de cálculo de los rendimientos.

Otra explicación posible para los altos rendimientos observados durante enero sería la llamada hipótesis de venta por motivos fiscales: en diciembre se venden activos que han tenido caídas en el mercado (reportando pérdidas al fisco) y se compran a inicios de enero (produciéndose un mercado alcista para el título). Los estudios de Reinganum (1983) y Branch (1977) sugieren tal posibilidad, pues en ambos casos se encontró que las acciones que declinaban pronunciadamente en diciembre producían rendimientos extraordinarios en enero del año siguiente, superando considerablemente al rendimiento promedio del mercado.

No obstante, se ha encontrado evidencia de que las estrategias de los inversionistas siguiendo criterios fiscales no explican necesariamente la presencia de rendimientos extraordinarios en enero. Por ejemplo, Jones, Pearce y Wilson (1987) estudiaron los rendimientos de 1821 a 1917 , periodo en el cual no existía todavía en Estados Unidos el impuesto a los ingresos, encontrando también evidencia empírica del efecto enero en los rendimientos y de magnitud comparable al observado en los estudios que lo han reportado para periodos posteriores en ese mercado.

Aunque no se ha resuelto la controversia respecto a su causa, se siguen reportando evidencias de la presencia del efecto enero en el mercado bursátil estadounidense, por ejemplo, Haug y Hirschey (2006) encuentran la presencia de tal efecto para periodos más recientes y confirman también la existencia del efecto enero como lo ha establecido la evidencia de la investigación previa. Sin embargo, de acuerdo con el análisis del índice Promedio Industrial del Dow Jones (DJIA) llevado a cabo por Moosa (2007), cubriendo el periodo 1970-2005, ha disminuido la importancia del efecto enero y los resultados de Moosa muestran la presencia de rendimientos extraordinarios en el mes de julio. Es decir, se estaría hablando entonces del surgimiento de otro patrón estacional el cual también habrá que considerar en estudios subsecuentes. 
Desde hace varios años se ha detectado también la presencia del efecto enero en mercados diferentes al de Estados Unidos. Gultekin y Gultekin (1983) extendieron el análisis a otros dieciséis mercados accionarios del mundo además del de Estados Unidos, observando también que en comparación con los otros meses del año en enero se observaban rendimientos más altos en la mayoría de los mercados analizados. En los estudios de Kato y Shallheim (1985) y Jaffe y Westerfield (1985) se reportan resultados similares en la bolsa de Tokio.

Es de destacarse que la investigación sobre la presencia del efecto enero en mercados emergentes es más reciente. Balaban (1995) estudia el mercado accionario turco y encuentra evidencia de que los rendimientos son más elevados en el mes de enero durante el periodo 1988-1993. Zang y Li (2006) estudian tres índices del mercado accionario de China, dando cuenta de la presencia del efecto enero en distintos periodos. Asteriou y Kavetsos (2006) buscan evidencia de la presencia de dicho efecto en los mercados accionarios de República Checa, Eslovaquia, Eslovena, Hungría, Lituania, Polonia, Rumanía y Rusia, durante el periodo de 1991 hasta principios de 2003. De acuerdo con sus resultados, existe evidencia estadísticamente significativa tanto del efecto enero como de efectos estacionales en esos mercados, siendo más fuerte dicha evidencia en los casos de los mercados de Hungría, Polonia y Rumanía.

Cabello y Ortiz (2003) reportan evidencia de un rendimiento positivo en enero durante el periodo 1986-2001 cuando analizan el comportamiento del mercado accionario mexicano, midiendo los rendimientos tanto en pesos mexicanos como en dólares de Estados Unidos. Sin embargo, al dividir la muestra en subperiodos esos investigadores encuentran que dicho efecto se desvanece tanto en los rendimientos medidos en la moneda local como en los rendimientos en dólares. Cabello y Ortiz (2004) extienden el análisis para detectar la presencia del efecto enero en los mercados de valores de Argentina, Brasil, Chile, Colombia, México, Perú y Venezuela, identificando el efecto enero en los mercados de Argentina, Chile y México. De manera un tanto sorpresiva, Cabello y Ortiz (2004) encuentran que precisamente en el mes de enero el mercado accionario de Perú ofrece los peores rendimientos del año.

Por otra parte, el efecto enero no es una anomalía exclusiva de los rendimientos de los mercados accionarios, como muestran Al-Khazali (2001) y Starks et al. (2006), quienes encuentran evidencia de este efecto para el mercado de bonos, y por Rendon y Ziemba (2007) para el mercado de futuros. No obstante, en este trabajo nos 
limitamos a analizar la presencia del efecto enero en los principales mercados accionarios de América Latina.

\section{Modelo econométrico}

Los rendimientos de los mercados accionarios se calcularon de la siguiente forma:

$$
R_{t}=\ln \left(P_{t} / P_{t-1}\right)
$$

donde $P_{t}$ es el valor al cierre mensual de cada uno de los índices accionarios para cada país y ln es el logaritmo natural.

Debido a que la prueba para el efecto enero se basa principalmente en el uso de variables dummy estacionales, se crearon 12 variables dummy estacionales (una para cada mes) que toman los siguientes valores:

$$
D_{i t}= \begin{cases}1 & \text { Si el rendimiento en el tiempo t corresponde al mes } i . \\ 0 & \text { de otra forma }\end{cases}
$$

Primero probamos la existencia de efectos estacionales en la serie de los rendimientos a través de la siguiente especificación:

$$
R_{t}=a_{1} D_{1 t}+a_{2} D_{2 t}+a_{3} D_{3 t}+\ldots+a_{12} D_{12 t}+u_{t}
$$

donde $R_{t}$ es el rendimiento en la mercado accionario en el tiempo $t, a_{i}$ es el rendimiento medio del mes i-ésimo, $D_{i t}$ son las variables dummy estacionales como se definió anteriormente, y $u_{t}$ es un término de error, idéntica e independientemente distribuido, iid. La hipótesis nula a ser probada es que los coeficientes $a_{i}$ son iguales. Si dichos coeficientes son iguales entonces no existen efectos estacionales, y viceversa.

De esta forma, para probar la presencia del efecto enero, el modelo de regresión se modifica de la siguiente manera:

$$
R_{t}=c+a_{2} D_{2 t}+a_{3} D_{3 t}+\ldots+a_{12} D_{12 t}+u_{t}
$$


bajo esta especificación, el intercepto $c$ representa el rendimiento medio en el mes de enero, y en este caso los coeficientes $a_{i}$, representan la diferencia entre el rendimiento de enero y el mes $i$.

La hipótesis nula a ser probada, bajo esta especificación, es que el resto de los coeficientes de las variables dummy son iguales a cero. Adicionalmente, valores negativos de los coeficientes dummy deben ser considerados como una prueba de la presencia del efecto enero. De esta manera, la estimación de los coeficientes en la ecuación (4) revela qué meses tienen rendimientos menores a los obtenidos en enero.

La varianza del modelo puede ser dependiente del tiempo y expresarse como una función de realizaciones pasadas. Bollerslev (1986) generalizó la metodología ARCH que introdujo Engle (1982). Se ha encontrado que este tipo de modelo es muy útil en economía y finanzas debido a su gran flexibilidad en la modelación del segundo momento de la distribución de los rendimientos. Si el término del proceso de error es:

$$
\begin{gathered}
\varepsilon_{t}=v_{t} \cdot \sqrt{h_{t}}, \text { donde } \sigma_{v}^{2}=1, E\left(v_{t}\right)=0 y \\
h_{t}=\alpha_{0}+\sum_{i=1}^{q} \alpha_{i} \varepsilon_{t-i}^{2}+\sum_{i=1}^{p} \beta_{i} h_{t-i}
\end{gathered}
$$

Entonces la secuencia $\left\{v_{t}\right\}$ es un proceso de ruido blanco y las medias condicional e incondicional de $\varepsilon_{t}$ son iguales a cero.

\section{Análisis empírico}

El periodo de estudio comprende datos mensuales de los índices elaborados por Morgan Stanley (MSCI) para los principales mercados accionarios de Latinoamérica. El periodo de análisis va de diciembre de 1987 a enero del 2009 para Argentina, Brasil, Chile y México, y de diciembre de 1992 a enero de 2009 para Colombia y Perú. De esta forma, se tienen 253 observaciones de los rendimientos para los primeros y 193 para los dos últimos. Con el fin de probar si los rendimientos de los países en cuestión presentan efectos estacionales se estimó la ecuación (3) cuando se calculan éstos tanto en su moneda local como en dólares. Los resultados de la estimación de la ecuación (3) se presentan en el cuadro 1, valuados en su propia moneda, y en el cuadro 5, valuados en dólares. 
La estrategia de estimación, para ambas pruebas, fue la siguiente: tanto para los rendimientos valuados en su moneda local o en dólares, si las ecuaciones estimadas para (3) y (4) presentan efectos ARCH, las especificaciones de ambas pruebas se modificaron para tener presente este tipo de efecto a través de un modelo GARCH y evaluar si bajo esta nueva especificación, la cual se considera más consistente, el efecto enero está presente o no.

Los resultados de las estimaciones efectuadas de la ecuación (3) para los rendimientos valuados en moneda local muestran que el mercado brasileño es el que muestra una mayor cantidad de efectos estacionales; por el contrario, el mercado accionario de Perú no presenta ningún efecto de este tipo como se puede apreciar en el cuadro 1. 


\section{Cuadro 1}

Resultados de la prueba de efectos estacionales en moneda local

\begin{tabular}{|c|c|c|c|c|c|c|}
\hline & Argentina & Brasil & Chile & Colombia & México & Perú \\
\hline \multirow[t]{2}{*}{$D_{1 t}$} & 0.0503 & 0.1474 & 0.0294 & 0.0352 & 0.0233 & 0.0130 \\
\hline & [0.264] & {$[0.000]$} & {$[0.030]$} & {$[0.090]$} & [0.184] & [0.576] \\
\hline \multirow[t]{2}{*}{$D_{2 t}$} & 0.0745 & 0.1156 & 0.0340 & -0.0063 & 0.0153 & 0.0337 \\
\hline & [0.106] & {$[0.004]$} & {$[0.015]$} & [0.767] & [0.393] & [0.161] \\
\hline \multirow[t]{2}{*}{$D_{3 t}$} & 0.0614 & 0.0500 & 0.0061 & -0.0147 & 0.0385 & 0.0162 \\
\hline & [0.183] & [0.203] & [0.657] & [0.491] & [0.033] & [0.501] \\
\hline \multirow[t]{2}{*}{$D_{4 t}$} & 0.0599 & 0.1041 & 0.0106 & 0.0497 & 0.0118 & 0.0329 \\
\hline & [0.193] & {$[0.008]$} & [0.444] & {$[0.021]$} & {$[0.510]$} & [0.172] \\
\hline \multirow[t]{2}{*}{$D_{5 t}$} & 0.0843 & 0.0799 & 0.0097 & -0.0069 & 0.0402 & 0.0103 \\
\hline & {$[0.068]$} & {$[0.043]$} & [0.485] & [0.747] & {$[0.026]$} & [0.669] \\
\hline \multirow[t]{2}{*}{$D_{6 t}$} & 0.0646 & 0.0307 & 0.0274 & -0.0173 & 0.0050 & 0.0120 \\
\hline & [0.161] & [0.435] & {$[0.048]$} & [0.418] & [0.779] & [0.617] \\
\hline \multirow[t]{2}{*}{$D_{7 t}$} & 0.0040 & 0.0870 & 0.0148 & 0.0225 & 0.0127 & -0.0017 \\
\hline & [0.930] & {$[0.027]$} & [0.285] & [0.294] & [0.480] & [0.945] \\
\hline \multirow[t]{2}{*}{$D_{8 t}$} & 0.0405 & 0.0378 & -0.0113 & -0.0062 & 0.0011 & 0.0004 \\
\hline & [0.379] & [0.336] & {$[0.415]$} & {$[0.770]$} & [0.951] & [0.987] \\
\hline \multirow[t]{2}{*}{$D_{9 t}$} & 0.0568 & 0.0845 & -0.0005 & 0.0207 & 0.0045 & 0.0267 \\
\hline & [0.217] & {$[0.032]$} & [0.972] & [0.333] & [0.802] & [0.267] \\
\hline \multirow[t]{2}{*}{$D_{10 t}$} & -0.0467 & 0.0427 & 0.0121 & -0.0043 & 0.0218 & -0.0237 \\
\hline & {$[0.31]$} & [0.278] & [0.381] & [0.839] & [0.224] & [0.323] \\
\hline \multirow[t]{2}{*}{$D_{11 t}$} & -0.0148 & 0.0734 & 0.0126 & 0.0442 & 0.0361 & 0.0067 \\
\hline & [0.747] & [0.062] & [0.364] & [0.039] & {$[0.045]$} & [0.780] \\
\hline \multirow[t]{2}{*}{$D_{12 t}$} & 0.1036 & 0.1468 & 0.0203 & 0.0456 & 0.0380 & 0.0285 \\
\hline & [0.025] & {$[0.000]$} & [0.142] & {$[0.034]$} & [0.035] & [0.235] \\
\hline$R^{2}$ & 0.08 & 0.21 & 0.08 & 0.10 & 0.09 & 0.05 \\
\hline \multirow[t]{2}{*}{$F$} & 1.77 & 5.51 & 1.80 & 1.73 & 1.94 & 0.73 \\
\hline & {$[0.054]$} & {$[0.000]$} & {$[0.050]$} & {$[0.064]$} & {$[0.030]$} & [0.719] \\
\hline D.W. & 1.66 & 1.43 & 1.69 & 1.69 & 1.93 & 1.96 \\
\hline \multirow[t]{2}{*}{$A R C H(1)$} & 36.15 & 36.15 & 0.08 & 2.26 & 11.18 & 1.68 \\
\hline & {$[0.000]$} & {$[0.000]$} & {$[0.775]$} & [0.133] & [0.001] & [0.195] \\
\hline$n$ & 253 & 253 & 253 & 253 & 253 & 193 \\
\hline
\end{tabular}

Nota: Los números entre corchetes son los valores $\mathrm{p}$ (p-values). 
Cuadro 2

Resultados de la prueba del efecto enero en moneda local

\begin{tabular}{|c|c|c|c|c|c|c|}
\hline & Argentina & Brasil & Chile & Colombia & Мéxico & Perú \\
\hline \multirow[t]{2}{*}{$c$} & 0.0503 & 0.1474 & 0.0294 & $\mathbf{0 . 0 3 5 2}$ & 0.0233 & 0.0130 \\
\hline & [0.264] & {$[0.000]$} & {$[0.030]$} & {$[0.090]$} & [0.184] & {$[0.576]$} \\
\hline \multirow[t]{2}{*}{$D_{2 t}$} & 0.0242 & -0.0318 & 0.0046 & -0.0415 & -0.0080 & 0.0207 \\
\hline & [0.707] & {$[0.562]$} & [0.813] & [0.164] & {$[0.750]$} & [0.536] \\
\hline \multirow[t]{2}{*}{$D_{3 t}$} & 0.0111 & -0.0974 & -0.0233 & -0.0499 & 0.0152 & 0.0031 \\
\hline & [0.863] & [0.077] & [0.230] & {$[0.094]$} & [0.545] & [0.925] \\
\hline \multirow[t]{2}{*}{$D_{4 t}$} & 0.0097 & -0.0433 & -0.0188 & 0.0145 & -0.0115 & 0.0198 \\
\hline & [0.881] & [0.430] & [0.331] & [0.626] & [0.647] & [0.553] \\
\hline \multirow[t]{2}{*}{$D_{5 t}$} & 0.0340 & -0.0675 & -0.0197 & -0.0421 & 0.0169 & -0.0028 \\
\hline & [0.596] & [0.219] & [0.308] & [0.158] & {$[0.501]$} & [0.934] \\
\hline \multirow[t]{2}{*}{$D_{6 t}$} & 0.0143 & -0.1167 & -0.0020 & -0.0525 & -0.0183 & -0.0010 \\
\hline & [0.824] & {$[0.034]$} & [0.919] & [0.079] & [0.466] & [0.976] \\
\hline \multirow[t]{2}{*}{$D_{7 t}$} & -0.0462 & -0.0604 & -0.0146 & -0.0127 & -0.0106 & -0.0147 \\
\hline & [0.472] & [0.272] & [0.451] & [0.668] & [0.671] & [0.661] \\
\hline \multirow[t]{2}{*}{$D_{8 t}$} & -0.0098 & -0.1096 & -0.0407 & -0.0414 & -0.0222 & -0.0126 \\
\hline & [0.879] & [0.047] & {$[0.036]$} & [0.165] & [0.376] & [0.706] \\
\hline \multirow[t]{2}{*}{$D_{9 t}$} & 0.0066 & -0.0629 & -0.0299 & -0.0145 & -0.0188 & 0.0137 \\
\hline & [0.919] & {$[0.252]$} & [0.123] & [0.626] & [0.453] & [0.683] \\
\hline \multirow[t]{2}{*}{$D_{10 t}$} & -0.0970 & -0.1048 & -0.0173 & -0.0395 & -0.0015 & -0.0368 \\
\hline & [0.132] & [0.057] & [0.372] & [0.185] & [0.954] & {$[0.272]$} \\
\hline \multirow[t]{2}{*}{$D_{11 t}$} & -0.0651 & -0.0740 & -0.0168 & 0.0090 & 0.0128 & -0.0063 \\
\hline & [0.312] & [0.178] & [0.384] & [0.762] & [0.608] & {$[0.85]$} \\
\hline \multirow[t]{2}{*}{$D_{12 t}$} & 0.0534 & -0.0007 & -0.0091 & 0.0104 & 0.0147 & 0.0155 \\
\hline & [0.407] & [0.990] & [0.640] & [0.726] & [0.557] & [0.643] \\
\hline$R^{2}$ & 0.04 & 0.05 & 0.04 & 0.08 & 0.03 & 0.03 \\
\hline \multirow[t]{2}{*}{$F$} & 0.89 & 1.04 & 0.86 & 1.44 & 0.65 & 0.48 \\
\hline & [0.554] & [0.415] & [0.577] & [0.160] & [0.780] & [0.914] \\
\hline D.W. & 1.66 & 1.43 & 1.69 & 1.69 & 1.93 & 1.96 \\
\hline \multirow[t]{2}{*}{$A R C H(1)$} & 36.15 & 21.26 & 0.08 & 2.26 & 11.18 & 1.68 \\
\hline & {$[0.000]$} & {$[0.000]$} & [0.775] & [0.133] & {$[0.001]$} & [0.195] \\
\hline$n$ & 253 & 253 & 253 & 193 & 253 & 193 \\
\hline
\end{tabular}

Nota: Los números entre corchetes son los valores p (p-values). 
Cuadro 3

Resultados de la prueba de efectos estacionales en moneda local con el modelo GARCH $(1,1)$

\begin{tabular}{|c|c|c|c|}
\hline & Argentina & Brasil & México \\
\hline \multirow[t]{2}{*}{$D_{1 t}$} & 0.0691 & 0.0460 & 0.0264 \\
\hline & {$[0.002]$} & {$[0.002]$} & {$[0.073]$} \\
\hline \multirow[t]{2}{*}{$D_{2 t}$} & 0.0066 & 0.0272 & 0.0084 \\
\hline & [0.743] & [0.197] & [0.648] \\
\hline \multirow[t]{2}{*}{$D_{3 t}$} & -0.0092 & 0.0050 & 0.0425 \\
\hline & [0.778] & [0.889] & {$[0.015]$} \\
\hline \multirow[t]{2}{*}{$D_{4 t}$} & 0.0091 & 0.0056 & 0.0028 \\
\hline & [0.683] & [0.784] & [0.889] \\
\hline \multirow[t]{2}{*}{$D_{5 t}$} & 0.0188 & 0.0304 & 0.0442 \\
\hline & [0.453] & [0.184] & {$[0.002]$} \\
\hline \multirow[t]{2}{*}{$D_{6 t}$} & 0.0146 & 0.0211 & 0.0050 \\
\hline & [0.613] & [0.314] & [0.765] \\
\hline \multirow[t]{2}{*}{$D_{7 t}$} & -0.0256 & 0.0031 & 0.0152 \\
\hline & [0.209] & [0.904] & [0.378] \\
\hline \multirow[t]{2}{*}{$D_{8 t}$} & 0.0016 & 0.0091 & 0.0046 \\
\hline & [0.954] & [0.643] & [0.779] \\
\hline \multirow[t]{2}{*}{$D_{9 t}$} & 0.0432 & 0.0217 & 0.0051 \\
\hline & {$[0.072]$} & [0.162] & {$[0.760]$} \\
\hline \multirow[t]{2}{*}{$D_{10 t}$} & -0.0215 & 0.0101 & 0.0236 \\
\hline & [0.367] & [0.628] & [0.110] \\
\hline \multirow[t]{2}{*}{$D_{11 t}$} & 0.0236 & 0.0329 & 0.0322 \\
\hline & [0.334] & [0.232] & {$[0.088]$} \\
\hline \multirow[t]{2}{*}{$D_{12 t}$} & 0.0759 & 0.0732 & 0.0428 \\
\hline & {$[0.004]$} & {$[0.021]$} & {$[0.039]$} \\
\hline \multirow[t]{2}{*}{$\alpha_{1}$} & 0.2642 & 0.2459 & 0.2482 \\
\hline & {$[0.000]$} & {$[0.001]$} & {$[0.025]$} \\
\hline \multirow[t]{2}{*}{$\beta_{1}$} & 0.7394 & 0.7571 & -0.2319 \\
\hline & {$[0.000]$} & {$[0.000]$} & {$[0.274]$} \\
\hline$n$ & 253 & 253 & 253 \\
\hline
\end{tabular}

Nota: Los números entre corchetes son los valores $\mathrm{p}$ (p-values) 
Cuadro 4

Resultados de la prueba del efecto enero en moneda local con el modelo GARCH $(1,1)$

\begin{tabular}{|c|c|c|c|}
\hline & Argentina & Brasil & México \\
\hline \multirow[t]{2}{*}{$c$} & 0.0691 & 0.0460 & 0.0264 \\
\hline & {$[0.002]$} & [0.002] & {$[0.073]$} \\
\hline \multirow[t]{2}{*}{$D_{2 t}$} & -0.0624 & -0.0188 & -0.0180 \\
\hline & [0.057] & {$[0.470]$} & [0.388] \\
\hline \multirow[t]{2}{*}{$D_{3 t}$} & -0.0783 & -0.0410 & 0.0160 \\
\hline & {$[0.055]$} & [0.279] & [0.471] \\
\hline \multirow[t]{2}{*}{$D_{4 t}$} & -0.0600 & -0.0404 & -0.0236 \\
\hline & {$[0.062]$} & {$[0.090]$} & [0.343] \\
\hline \multirow[t]{2}{*}{$D_{5 t}$} & -0.0502 & -0.0156 & 0.0177 \\
\hline & [0.139] & [0.576] & [0.391] \\
\hline \multirow[t]{2}{*}{$D_{6 t}$} & -0.0545 & -0.0249 & -0.0214 \\
\hline & [0.129] & [0.328] & [0.336] \\
\hline \multirow[t]{2}{*}{$D_{7 t}$} & -0.0947 & -0.0429 & -0.0113 \\
\hline & {$[0.002]$} & [0.147] & [0.611] \\
\hline \multirow[t]{2}{*}{$D_{8 t}$} & -0.0675 & -0.0369 & -0.0218 \\
\hline & {$[0.038]$} & [0.136] & [0.302] \\
\hline \multirow[t]{2}{*}{$D_{9 t}$} & -0.0259 & -0.0243 & -0.0213 \\
\hline & {$[0.430]$} & [0.268] & [0.341] \\
\hline \multirow[t]{2}{*}{$D_{10 t}$} & -0.0905 & -0.0359 & -0.0028 \\
\hline & {$[0.006]$} & [0.169] & [0.891] \\
\hline \multirow[t]{2}{*}{$D_{11 t}$} & -0.0455 & -0.0131 & 0.0058 \\
\hline & [0.195] & [0.661] & [0.807] \\
\hline \multirow[t]{2}{*}{$D_{12 t}$} & 0.0069 & 0.0272 & 0.0163 \\
\hline & [0.847] & [0.417] & [0.530] \\
\hline \multirow[t]{2}{*}{$\alpha_{1}$} & 0.2642 & 0.2459 & 0.2482 \\
\hline & {$[0.000]$} & {$[0.001]$} & {$[0.025]$} \\
\hline \multirow[t]{2}{*}{$\beta_{1}$} & 0.7394 & 0.7571 & -0.2319 \\
\hline & {$[0.000]$} & {$[0.000]$} & [0.274] \\
\hline$n$ & 253 & 253 & 253 \\
\hline
\end{tabular}

Nota: Los números entre corchetes son los valores p (p-values) 


\section{Cuadro 5}

Resultados de la prueba de efectos estacionales en dólares

\begin{tabular}{|c|c|c|c|c|c|c|}
\hline & Argentina & Brasil & Chile & Colombia & México & Perú \\
\hline \multirow[t]{2}{*}{$D_{1 t}$} & -0.0072 & 0.0345 & 0.0284 & 0.0174 & 0.0109 & 0.0053 \\
\hline & [0.820] & [0.319] & [0.067] & [0.446] & [0.598] & [0.824] \\
\hline \multirow[t]{2}{*}{$D_{2 t}$} & -0.0154 & 0.0495 & 0.0372 & -0.0031 & 0.0093 & 0.0297 \\
\hline & [0.636] & [0.162] & [0.019] & [0.894] & {$[0.660]$} & [0.227] \\
\hline \multirow[t]{2}{*}{$D_{3 t}$} & 0.0344 & -0.0180 & 0.0028 & -0.0178 & 0.0329 & 0.0151 \\
\hline & [0.291] & [0.612] & [0.858] & {$[0.450]$} & [0.121] & [0.539] \\
\hline \multirow{2}{*}{$D_{4 t}$} & 0.0281 & 0.0595 & 0.0136 & 0.0460 & 0.0203 & 0.0285 \\
\hline & [0.388] & [0.094] & [0.391] & {$[0.052]$} & [0.339] & [0.246] \\
\hline \multirow{2}{*}{$D_{5 t}$} & 0.0271 & 0.0103 & 0.0010 & -0.0124 & 0.0307 & 0.0057 \\
\hline & [0.405] & [0.770] & [0.948] & [0.600] & [0.148] & [0.815] \\
\hline \multirow[t]{2}{*}{$D_{6 t}$} & -0.0025 & -0.0372 & 0.0109 & -0.0295 & 0.0002 & 0.0097 \\
\hline & [0.939] & [0.293] & [0.489] & [0.213] & [0.992] & [0.692] \\
\hline \multirow[t]{2}{*}{$D_{7 t}$} & -0.0144 & 0.0126 & 0.0116 & 0.0193 & 0.0185 & -0.0005 \\
\hline & {$[0.658]$} & [0.722] & [0.462] & [0.413] & {$[0.383]$} & [0.984] \\
\hline \multirow[t]{2}{*}{$D_{8 t}$} & 0.0282 & -0.0147 & -0.0153 & -0.0292 & -0.0108 & -0.0078 \\
\hline & [0.386] & [0.679] & [0.333] & [0.217] & [0.610] & [0.751] \\
\hline \multirow[t]{2}{*}{$D_{9 t}$} & 0.0616 & -0.0008 & -0.0025 & -0.0032 & -0.0054 & 0.0227 \\
\hline & [0.059] & [0.982] & [0.875] & [0.891] & [0.798] & [0.355] \\
\hline \multirow[t]{2}{*}{$D_{10 t}$} & -0.0561 & -0.0319 & -0.0014 & -0.0028 & 0.0035 & -0.0269 \\
\hline & [0.085] & [0.367] & [0.928] & [0.906] & [0.868] & [0.273] \\
\hline \multirow[t]{2}{*}{$D_{11 t}$} & -0.0303 & -0.0079 & 0.0057 & 0.0507 & 0.0335 & 0.0056 \\
\hline & {$[0.352]$} & [0.822] & [0.719] & [0.033] & [0.114] & [0.818] \\
\hline \multirow[t]{2}{*}{$D_{12 t}$} & 0.0672 & 0.0777 & 0.0269 & 0.0532 & 0.0167 & 0.0276 \\
\hline & {$[0.040]$} & [0.029] & {$[0.090]$} & {$[0.025]$} & [0.431] & [0.261] \\
\hline$R^{2}$ & 0.06 & 0.05 & 0.06 & 0.09 & 0.04 & 0.04 \\
\hline \multirow[t]{2}{*}{$F$} & 1.29 & 1.10 & 1.23 & 1.57 & 0.85 & 0.58 \\
\hline & [0.223] & [0.357] & [0.266] & [0.105] & {$[0.601]$} & [0.857] \\
\hline D.W. & 1.85 & 2.22 & 1.68 & 1.59 & 1.77 & 1.92 \\
\hline \multirow[t]{2}{*}{$A R C H(1)$} & 3.91 & 8.06 & 0.01 & 0.41 & 8.15 & 1.24 \\
\hline & {$[0.048]$} & {$[0.005]$} & [0.921] & {$[0.520]$} & {$[0.004]$} & [0.265] \\
\hline$n$ & 253 & 253 & 253 & 193 & 253 & 193 \\
\hline
\end{tabular}

Nota: Los números entre corchetes son los valores p (p-values). 


\section{Cuadro 6}

\section{Resultados de la prueba del efecto enero en dólares}

\begin{tabular}{|c|c|c|c|c|c|c|}
\hline & Argentina & Brasil & Chile & Colombia & México & Perú \\
\hline \multirow[t]{2}{*}{$c$} & -0.0072 & 0.0345 & 0.0284 & 0.0174 & 0.0109 & 0.0053 \\
\hline & {$[0.820]$} & [0.319] & [0.067] & [0.446] & [0.598] & [0.824] \\
\hline \multirow[t]{2}{*}{$D_{2 t}$} & -0.0081 & 0.0150 & 0.0087 & -0.0206 & -0.0016 & 0.0244 \\
\hline & [0.858] & [0.761] & [0.693] & [0.531] & [0.958] & [0.475] \\
\hline \multirow[t]{2}{*}{$D_{3 t}$} & 0.0417 & -0.0524 & -0.0256 & -0.0353 & 0.0220 & 0.0098 \\
\hline & [0.360] & [0.290] & [0.248] & [0.284] & [0.457] & [0.774] \\
\hline \multirow[t]{2}{*}{$D_{4 t}$} & 0.0353 & 0.0250 & -0.0149 & 0.0285 & 0.0094 & 0.0232 \\
\hline & [0.438] & [0.614] & [0.502] & [0.386] & [0.752] & [0.497] \\
\hline \multirow[t]{2}{*}{$D_{5 t}$} & 0.0343 & -0.0241 & -0.0274 & -0.0298 & 0.0198 & 0.0004 \\
\hline & {$[0.450]$} & {$[0.625]$} & {$[0.216]$} & {$[0.365]$} & {$[0.504]$} & [0.989] \\
\hline \multirow[t]{2}{*}{$D_{6 t}$} & 0.0047 & -0.0717 & -0.0175 & -0.0469 & -0.0107 & 0.0044 \\
\hline & [0.917] & [0.148] & [0.430] & [0.155] & [0.718] & [0.897] \\
\hline \multirow[t]{2}{*}{$D_{7 t}$} & -0.0071 & -0.0219 & -0.0168 & 0.0019 & 0.0076 & -0.0058 \\
\hline & {$[0.875]$} & [0.658] & [0.448] & [0.954] & [0.797] & [0.866] \\
\hline \multirow[t]{2}{*}{$D_{8 t}$} & 0.0355 & -0.0491 & -0.0437 & -0.0466 & -0.0217 & -0.0131 \\
\hline & [0.436] & [0.321] & [0.049] & [0.157] & [0.464] & [0.702] \\
\hline \multirow[t]{2}{*}{$D_{9 t}$} & 0.0689 & -0.0353 & -0.0309 & -0.0207 & -0.0163 & 0.0175 \\
\hline & [0.131] & [0.476] & [0.163] & [0.529] & [0.582] & [0.610] \\
\hline \multirow[t]{2}{*}{$D_{10 t}$} & -0.0489 & -0.0664 & -0.0298 & -0.0202 & -0.0074 & -0.0322 \\
\hline & [0.283] & [0.180] & [0.178] & [0.539] & [0.804] & [0.346] \\
\hline \multirow[t]{2}{*}{$D_{11 t}$} & -0.0230 & -0.0424 & -0.0227 & 0.0332 & 0.0226 & 0.0004 \\
\hline & [0.613] & [0.391] & [0.304] & [0.313] & [0.445] & [0.992] \\
\hline \multirow[t]{2}{*}{$D_{12 t}$} & 0.0744 & 0.0433 & -0.0015 & 0.0358 & 0.0058 & 0.0223 \\
\hline & [0.103] & [0.382] & [0.944] & [0.277] & [0.845] & [0.514] \\
\hline$R^{2}$ & 0.06 & 0.05 & 0.04 & 0.09 & 0.02 & 0.03 \\
\hline \multirow[t]{2}{*}{$F$} & 1.31 & 1.09 & 0.90 & 1.60 & 0.49 & 0.47 \\
\hline & {$[0.221]$} & [0.367] & [0.541] & [0.102] & [0.910] & [0.922] \\
\hline D.W. & 1.85 & 2.22 & 1.68 & 1.59 & 1.77 & 1.92 \\
\hline \multirow[t]{2}{*}{$A R C H(1)$} & 3.91 & 8.06 & 0.01 & 0.41 & 8.15 & 1.24 \\
\hline & {$[0.048]$} & {$[0.005]$} & [0.921] & [0.520] & {$[0.004]$} & [0.265] \\
\hline$n$ & 253 & 253 & 253 & 193 & 253 & 193 \\
\hline
\end{tabular}

Nota: Los números entre corchetes son los valores $\mathrm{p}$ (p-values). 


\section{Cuadro 7}

Resultados de la prueba de efectos estacionales en dólares con el modelo GARCH $(1,1)$

\begin{tabular}{|c|c|c|c|}
\hline & Argentina & Brasil & México \\
\hline \multirow[t]{2}{*}{$D_{1 t}$} & 0.0447 & 0.0311 & 0.0283 \\
\hline & [0.094] & [0.329] & [0.183] \\
\hline \multirow[t]{2}{*}{$D_{2 t}$} & -0.0078 & 0.0388 & 0.0108 \\
\hline & {$[0.721]$} & [0.448] & [0.627] \\
\hline \multirow[t]{2}{*}{$D_{3 t}$} & 0.0047 & -0.0198 & 0.0360 \\
\hline & [0.869] & [0.520] & [0.082] \\
\hline \multirow[t]{2}{*}{$D_{4 t}$} & 0.0314 & 0.0340 & 0.0167 \\
\hline & {$[0.272]$} & [0.430] & [0.490] \\
\hline \multirow[t]{2}{*}{$D_{5 t}$} & 0.0200 & 0.0179 & 0.0357 \\
\hline & [0.388] & [0.675] & [0.029] \\
\hline \multirow[t]{2}{*}{$D_{6 t}$} & 0.0060 & -0.0329 & 0.0072 \\
\hline & [0.855] & [0.338] & [0.718] \\
\hline \multirow[t]{2}{*}{$D_{7 t}$} & -0.0221 & 0.0024 & 0.0160 \\
\hline & [0.344] & [0.956] & [0.507] \\
\hline \multirow[t]{2}{*}{$D_{8 t}$} & 0.0023 & -0.0218 & -0.0030 \\
\hline & [0.934] & [0.533] & [0.901] \\
\hline \multirow[t]{2}{*}{$D_{9 t}$} & 0.0447 & 0.0069 & 0.0011 \\
\hline & [0.077] & [0.851] & [0.952] \\
\hline \multirow[t]{2}{*}{$D_{10 t}$} & -0.0256 & -0.0344 & 0.0164 \\
\hline & [0.334] & [0.363] & [0.394] \\
\hline \multirow[t]{2}{*}{$D_{11 t}$} & 0.0151 & -0.0046 & 0.0377 \\
\hline & [0.619] & [0.919] & [0.150] \\
\hline \multirow[t]{2}{*}{$D_{12 t}$} & 0.0740 & 0.0767 & 0.0147 \\
\hline & [0.007] & [0.065] & [0.481] \\
\hline \multirow[t]{2}{*}{$\alpha_{1}$} & 0.1903 & 0.1146 & 0.1313 \\
\hline & {$[0.000]$} & [0.203] & {$[0.081]$} \\
\hline \multirow[t]{2}{*}{$\beta_{1}$} & 0.7875 & -0.2141 & 0.7382 \\
\hline & {$[0.000]$} & [0.632] & {$[0.000]$} \\
\hline$n$ & 253 & 253 & 253 \\
\hline
\end{tabular}

Nota: Los números entre corchetes son los valores p (p-values). 


\section{Cuadro 8}

Resultados de la prueba del efecto enero en dólares con el modelo GARCH $(1,1)$

\begin{tabular}{|c|c|c|c|}
\hline & Argentina & Brasil & México \\
\hline \multirow[t]{2}{*}{$c$} & 0.0447 & 0.0311 & 0.0283 \\
\hline & {$[0.094]$} & [0.329] & [0.183] \\
\hline \multirow{2}{*}{$D_{2 t}$} & -0.0525 & 0.0077 & -0.0175 \\
\hline & {$[0.134]$} & [0.899] & [0.552] \\
\hline \multirow[t]{2}{*}{$D_{3 t}$} & -0.0400 & -0.0509 & 0.0078 \\
\hline & [0.309] & [0.263] & [0.793] \\
\hline \multirow[t]{2}{*}{$D_{4 t}$} & -0.0134 & 0.0029 & -0.0116 \\
\hline & [0.742] & [0.957] & [0.721] \\
\hline \multirow{2}{*}{$D_{5 t}$} & -0.0247 & -0.0132 & 0.0074 \\
\hline & {$[0.501]$} & [0.805] & [0.779] \\
\hline \multirow[t]{2}{*}{$D_{6 t}$} & -0.0387 & -0.0640 & -0.0210 \\
\hline & [0.356] & {$[0.165]$} & [0.483] \\
\hline \multirow[t]{2}{*}{$D_{7 t}$} & -0.0669 & -0.0287 & -0.0123 \\
\hline & {$[0.064]$} & [0.596] & [0.705] \\
\hline \multirow[t]{2}{*}{$D_{8 t}$} & -0.0424 & -0.0529 & -0.0313 \\
\hline & [0.269] & {$[0.267]$} & [0.321] \\
\hline \multirow[t]{2}{*}{$D_{9 t}$} & 0.0000 & -0.0242 & -0.0272 \\
\hline & [1.000] & [0.617] & [0.348] \\
\hline \multirow[t]{2}{*}{$D_{10 t}$} & -0.0704 & -0.0655 & -0.0118 \\
\hline & [0.073] & [0.186] & [0.666] \\
\hline \multirow[t]{2}{*}{$D_{11 t}$} & -0.0296 & -0.0357 & 0.0095 \\
\hline & [0.490] & {$[0.522]$} & [0.781] \\
\hline \multirow[t]{2}{*}{$D_{12 t}$} & 0.0293 & 0.0456 & -0.0136 \\
\hline & [0.463] & [0.381] & [0.655] \\
\hline \multirow[t]{2}{*}{$\alpha_{1}$} & 0.1903 & 0.1146 & 0.1313 \\
\hline & {$[0.000]$} & [0.203] & {$[0.081]$} \\
\hline \multirow[t]{2}{*}{$\beta_{1}$} & 0.7875 & -0.2141 & 0.7382 \\
\hline & {$[0.000]$} & [0.632] & [0.000] \\
\hline$n$ & 253 & 253 & 253 \\
\hline
\end{tabular}

Nota: Los números entre corchetes son los valores $\mathrm{p}$ (p-values). 
Sin embargo, la estimación para el mercado accionario de Brasil presenta efectos $\mathrm{ARCH}$, por lo que se procedió a realizar la estimación a través del modelo GARCH(1,1). Se observa que con la especificación para incluir los efectos $\mathrm{ARCH}$ disminuye considerablemente la presencia de efectos estacionales significativos en el mercado brasileño, como se puede ver en el cuadro 3 en el que se muestran en negritas los coeficientes estadísticamente significativos con sus correspondientes valores $p$ encerrados en corchetes.

Las estimaciones de la ecuación (4) para evaluar la presencia o no del efecto enero en los rendimientos de estos mercados valuados en su propia moneda se presentan en el cuadro 2, las cuales dan cuenta de que dicho efecto está presente en los mercados de Brasil, Chile y Colombia, aunque este último al nivel de significancia del 10\%. No obstante, la estimación para los rendimientos del mercado de Brasil presenta efectos ARCH al igual que para los mercado de Argentina y México por lo que dicha especificación se volvió a estimar a través del modelo GARCH(1,1), bajo esta especificación los mercados de Argentina, Brasil y México presentan efectos ARCH.

Como se mencionó anteriormente, la prueba de la presencia del efecto enero requiere, además de que el coeficiente que trata de capturar los rendimientos en dicho mes sea significativo en (4), lo cual puede ser una condición débil, que el resto de los coeficientes sean nulos, esto último puede constituir una condición fuerte. Por esta razón, se realizaron pruebas de Wald a las estimaciones realizadas de (4) que no presentaron efectos ARCH como a las que se llevaron a cabo estimando la ecuación (5) en forma conjunta. Los resultados de las prueba para los rendimientos valuados en moneda local y en dólares se presentan en los cuadro 9 y 10 respectivamente.

\section{Cuadro 9}

Prueba de Wald para los modelos estimados con rendimientos valuados en moneda local $\left(H_{0}: a_{2}=a_{3}=\ldots=a_{12}=0\right)$

\begin{tabular}{cccc}
\hline País & Modelo & Wald & Valor-p \\
\hline Argentina & GARCH & $\chi^{2}(11)=23.17$ & {$[0.0167]$} \\
Brasil & GARCH & $\chi^{2}(11)=7.57$ & {$[0.7509]$} \\
Chile & MCO & $F(11,241)=0.86$ & {$[0.5767]$} \\
Colombia & MCO & $F(11,181)=1.44$ & {$[0.1597]$} \\
México & GARCH & $\chi^{2}(11)=10.82$ & {$[0.4581]$} \\
\hline
\end{tabular}




\section{Cuadro 10}

Prueba de Wald para los modelos estimados con rendimientos valuados en dólares

$$
\left(H_{0}: a_{2}=a_{3}=\ldots=a_{12}=0\right)
$$

\begin{tabular}{cccc}
\hline País & Modelo & Wald & Valor-p \\
& & & \\
Argentina & GARCH & $\chi^{2}(11)=16.59$ & {$[0.1208]$} \\
Chile & MCO & $F(11,241)=0.90$ & {$[0.5408]$} \\
& & & \\
\hline
\end{tabular}

A partir de las pruebas de Wald, efectuadas a los modelos, se corrobora la "condición fuerte" del efecto enero para todos los mercados, con excepción de Argentina cuando los rendimientos están valuados en moneda local. En este último mercado sólo se cumple lo que hemos denominado la "condición débil"; es decir, el coeficiente que mide el rendimiento promedio en dicho mes es significativo, sin embargo, se rechaza la hipótesis nula de que el resto de los coeficientes de los otros meses sea igual a cero,

$$
\left(H_{0}: a_{2}=a_{3}=\ldots=a_{12}=0\right) .
$$

De esta manera se verifica que casi todos los mercados considerados, con excepción de Perú, presentan el efecto enero cuando la prueba se realiza sobre los rendimientos del mercado valuados en su respectiva moneda. Mientras que cuando las pruebas se efectuaron sobre los rendimientos valuados en dólares, únicamente los mercados de Argentina y Chile dan cuenta de la presencia de este tipo de efecto. No obstante, las estimaciones para Argentina, Brasil y México presentaron efectos ARCH por lo que se volvió a efectuar la estimación de la estimación (4) teniendo presente el efecto ARCH detectado. Los resultados de esta última especificación se muestran en el cusadro 8, de los cuales solamente Argentina y Chile revelan la presencia del efecto enero, resultado que se verifica con la prueba de Wald porque no es posible rechazar la hipótesis nula de que el resto de los coeficientes es igual a cero para estos países, como se muestra en el cuadro 10.

\section{Conclusiones}

Se examinó la posible existencia de efectos estacionales y del "efecto enero" en los rendimientos accionarios de los principales mercados de Latinoamérica. A grandes 
rasgos encontramos evidencia mixta sobre la presencia del efecto enero en estos mercados. Cuando la prueba se realiza sobre los rendimientos valuados en moneda local la mayoría de los mercados presentan evidencia de dicho efecto, pues las estimaciones sugieren su presencia en los mercados de Argentina, Brasil, Colombia, Chile y México, mientras que cuando la prueba se realiza sobre los rendimientos valuados en dólares sólo Argentina y Chile ofrecen evidencia de tal efecto. Las estimaciones efectuadas no dan cuenta de la existencia del efecto enero para el mercado de Perú, ni cuando se valúan los rendimientos en moneda local ni cuando se valúan en dólares. Los únicos mercados accionarios que presentan dicho efecto en sus rendimientos, valuados tanto en moneda local como en dólares, son los de Argentina y Chile.

Por otra parte, el análisis efectuado en las páginas previas sugiere la importancia de especificar correctamente las pruebas para detectar efectos estacionales en el comportamiento de las series de mercados accionarios; es decir, en el modelado para las pruebas correspondientes se deben tomar en cuenta las características propias de la series analizadas. Como se desprende de los resultados que se han reportado en páginas anteriores, para llevar a cabo las pruebas de efectos estacionales y del efecto enero es muy importante considerar la presencia de efectos ARCH (típicos en los rendimientos financieros), pues al tomarlos en cuenta se pueden modificar sustancialmente las conclusiones de tales pruebas.

También es conveniente destacar que los resultados reportados en este trabajo muestran que la forma en como se valúen los rendimientos, ya sea en monedas locales o en una moneda común como el dólar, es fundamental para detectar o no la presencia del efecto enero y de otros efectos estacionales. Naturalmente, lo anterior es equivalente a reconocer que la dinámica del tipo de cambio durante el periodo de estudio juega un papel preponderante para determinar los resultados de las pruebas empíricas.

Los resultados del presente estudio tienen implicaciones también para los inversionistas de los países analizados y los inversionistas extranjeros. No obstante el grado de apertura existente en los principales mercados latinoamericanos como consecuencia de sus procesos de liberalización, los resultados de esta investigación sugieren que no están operando aún las condiciones de arbitraje suficientes para eliminar la posibilidad de rendimientos extraordinarios en ellos de acuerdo con efectos de calendario. En última instancia, la existencia de dicha posibilidad puede permitir que se instrumenten estrategias de inversión para explotarla. 
La literatura previa ha pasado por alto la cuestión de que los resultados pueden ser diferentes para un mismo mercado, dependiendo de que si los rendimientos se miden en la moneda local o en dólares. Sin duda, lo que está detrás de esta discrepancia en estos resultados es la incidencia del tipo de cambio sobre los rendimientos accionarios y posiblemente la forma en que se interrelacionan los mercados cambiarios y accionarios; lo cual requiere una mayor investigación futura. Esto pone también de manifiesto la importancia que tiene el riesgo cambiario para los tomadores de decisiones de inversión de portafolios que buscan obtener rendimientos extraordinarios o anormales con base en reglas como el explotar el "efecto enero".

\section{Referencias}

Al-Khazali, O. M. (2001). "Does the January Effect exist in High-Yield Bond Market?" Review of Financial Economics, 10, 71-80.

Asteriou, D. y George K. (2006). "Testing for the Existence of the 'January Effect' in Transition Economies". Applied Financial Economics Letters, 2 (6), 375-382

Balaban, E. (1995). "January effect, yes! What about Mark Twain effect?" Discussion paper 9509, The Central Bank of the Republic of Turkey, Research Deparment.

Blume, M. E. y Robert F. S. (1983). "Biased in computed returns". Journal of Financial Economics 12, 387-404.

BollersLev, T. (1986). "Generalized autoregressive conditional heteroskedasticity". Journal of Econometrics, 31 (3), 307-327.

BRANCH, B. (1977). “A tax-loss trading rule”. Journal of Business, 50, 198-207.

Cabello, Alejandra y Edgar Ortiz (2003). "Day of the week and month of the year anomalies in the Mexican stock market". Revista Mexicana de Economía y Finanzas 2 (3), 217-241.

(2004). "Day of the week and month of the year effects at the Latin American emerging markets". International Finance Review 5, 273-304. 
ENGLE, R. F. (1982). “Autoregressive conditional heteroscedasticity with estimates of the variance of United Kingdom inflation". Econometrica, 50, 9871007.

FAMA, E. F. (1991). "Efficient capital markets: II". Journal of Finance, 46 (5), 1575-1617.

Gultekin, M. N. y N. B. GulteKIN (1983). "Stock market seasonality: international evidence". Journal of Financial Economics, 12 (4), 469-481.

Haugen, R. A. y J. LaKonishoK (1988). The incredible January Effect: The stock market's unsolved mystery, Dow-Jones-Irwin, Homewood, IL.

HaI, L. y M. QIngZhong (2003). "Do Earnings Explain the January Effect". Working Paper. University of Southern California. Social Science Research Network.

Haug, M. y M. Hirschey (2006). "The January effect". Financial Analyst Journal, 62 (5), 78-88.

JEFFREY, J. y R. WESTERFIELD (1985). "Patterns in Japanese common stock returns: day of the week and turn of the year effects". Journal of Financial and Quantitative Analysis, 20 (2), 261-272.

Jones, Ch. P., D. K. Pearce y J. W. Wilson (1987). "Can Tax-Loss Selling Explain the January Effect? A Note". Journal of Finance, 42 (2), 453-461.

Kato, K. y J. S. Shallheim (1985). "Seasonal and size anomalies in the Japanese stock market". Journal of Financial and Quantitative Analysis, 20 (2), 243260

KeIm, D. B. (1983). "Size-related anomalies and stock return seasonality: Further empirical evidence". Journal of Financial Economics, 12 (1), 13-32.

KeIM, D. B. (1989). "Trading patterns, bid-ask spreads, and estimated security returns : The case of common stocks at calendar turning points". Journal of Financial Economics, 25 (1), 75-97. 
MoosA, I. A. (2007). "The vanishing January effect". International Research Journal of Finance and Economics, 7, 92-103.

RENDON, J. y W.T. ZiEmbA, (2007). "Is the January effect still alive in the futures markets?" Financial Markets and Portfolio Management, 21 (3), 381-396.

Reinganum, M. R. (1983). "The anomalous stock market behavior of small firms in January: Empirical tests for tax-loss selling effects". Journal of Financial Economics, 12 (1), 89-104.

RozefF, M. S. y W. R. KInNey JR. (1976). "Capital market seasonality: The case of stock returns". Journal of Financial Economics 3, 379-402.

Starks, L.T., Yong, L. y L. Zheng (2006). "Tax-Loss Selling and the January Effect: Evidence from Municipal Bond Closed-End Funds". The Journal of Finance, 61 (6), 3049-3067.

Zhang, B. y X. Li (2006). "Do Calendar Effects Still Exist in the Chinese Stock Markets?" Journal of Chinese Economic and Business Studies, 4 (2) 151-163 